\title{
Finite-amplitude bifurcations in plane Poiseuille flow: two-dimensional Hopf bifurcation
}

\author{
By ISRAEL SOIBELMAN AND DANIEL I. MEIRON \\ Applied Mathematics, California Institute of Technology, Pasadena, CA 91125, USA
}

(Received 13 March 1990 and in revised form 21 January 1991)

We examine the stability to superharmonic disturbances of finite-amplitude twodimensional travelling waves of permanent form in plane Poiseuille flow. The stability characteristics of these flows depend on whether the flux or pressure gradient are held constant. For both conditions we find several Hopf bifurcations on the upper branch of the solution surface of these two-dimensional waves. We calculate the periodic orbits which emanate from these bifurcations and find that there exist no solutions of this type at Reynolds numbers lower than the critical value for existence of two-dimensional waves $(\approx 2900)$. We confirm the results of Jiménez (1987) who first detected a stable branch of these solutions by integrating the two-dimensional equations of motion numerically.

\section{Introduction}

A major area of research in fluid mechanics is the transition of laminar plane Poiseuille flow to turbulent flow. Plane Poiseuille flow is the viscous incompressible flow between two parallel surfaces driven by a streamwise pressure gradient. The basic laminar solution for the velocity field is the parabolic profile

$$
u=U_{0}\left[1-\left(\frac{y}{h}\right)^{2}\right]
$$

A linear analysis is the natural first step in studying stability of the basic flow. The governing equation for infinitesimal disturbances of the form

$$
u^{\prime}=\hat{u}(y) \mathrm{e}^{\mathrm{i} \alpha(x-c t)}
$$

where $\alpha$ is the wavenumber and $c$ is the complex phase speed, is the Orr $\rightarrow$ Sommerfeld equation. Only one eigenfunction of the Orr-Sommerfeld equation is known to have an eigenvalue whose imaginary part, $c_{i}(\alpha, R e)$, becomes greater than zero in a region of the $(\alpha, R e)$-plane. The curve of marginal stability, $c_{i}=0$, is known as the Orr-Sommerfeld neutral curve, and the critical Reynolds number of this curve is 5772.22 .

The critical Reynolds number from linear theory does not coincide with the experimentally observed critical Reynolds number when finite-amplitude disturbances are considered. The experiments of Carlson, Widnall \& Peeters (1982), Nishioka \& Asai (1985) and Alavyoon, Henningson \& Alfredsson (1986) all indicate that transition occurs at a Reynolds number of $\approx 1000$, where the Reynolds number is defined in terms of the centreline velocity and half-height of the channel. This result suggests that finite-amplitude disturbances initiate transition. This conclusion 
is also supported by observations from plane Couette flow. Plane Couette flow is the viscous incompressible flow between two parallel surfaces driven by the upper surface. The basic laminar solution for the velocity field is

$$
u=U_{0} \frac{y}{h} .
$$

Plane Couette flow is believed to be linearly stable for all Reynolds number (Drazin \& Reid 1981). The experiments by Reichardt (1956) and others however, suggest that the transition to turbulence is very much like that of plane Poiseuille flow and occurs at a Reynolds number of $\approx 750$. Clearly, finite-amplitude disturbances need to be investigated to understand transition.

In the literature, there are two general approaches to studying finite-amplitude disturbances. One approach utilizes a numerical simulation of the time dependence of the disturbance. This approach has been used by Orszag \& Kells (1980), Orszag \& Patera (1983), Kleiser (1982), Kim (1983), and others. The advantages to this approach include the feasibility of high-resolution numerics, and the ability to simulate experimentally observed flows. The disadvantages include the inability to calculate unstable solutions, the lack of control over the solution form, and the difficulty in implementing a parameter search of steady solutions.

An alternative approach, which we use in this paper, is to search for specific types of steady equilibrium solutions. This approach is capable of computing unstable solutions. The solution form can also be specified, and a parameter search can be undertaken in solution space. A major disadvantage to this approach is that highresolution numerics have not been feasible. As will be shown in $\$ 2$, however, with spectral methods calculations performed with a few modes are often sufficient to give good qualitative and sometimes quantitative results.

Our approach to studying finite-amplitude disturbances is based on Saffman's (1983) hypothesis that the transition from the laminar state to the turbulent state is dependent on the existence of intermediate vortical states and that the transition to turbulence is the three-dimensional instability of these states and their complex interactions. Typical examples of vortical states are spatially or temporally periodic solutions. The exact nature of the vortical state remains to be determined. Among the many possibilities are two-dimensional travelling waves, quasi-periodic solutions, and three-dimensional waves. Although the form of the vortical solution is not specified, the solution should exist at Reynolds number lower than the critical Reynolds number of linear theory, and the instabilities of the solution should grow on the convective timescale observed in experiment.

Perhaps the simplest example of vortical states are two-dimensional travelling waves. Chen \& Joseph (1973) proved the existence of two-dimensional travelling waves which bifurcate from plane Poiseuille flow. The first attempt to compute these waves was undertaken by Noether (1921), who studied equilibrium wave disturbances by expanding the disturbance in a Fourier series and truncating the resulting equations by using only one Fourier mode in the periodic direction. This is the 'mean-field' approximation and only takes into account the correction to the mean flow and the first harmonic; no higher harmonics are included. Using asymptotic expansions, Meksyn \& Stuart (1951) obtained an approximate solution for these equations. They found that the critical Reynolds number decreases with increasing disturbance amplitude, eventually passes through a limit point and increases. The critical two-dimensional Reynolds number was found to be at a wavenumber larger than the maximum critical wavenumber on the Orr-Sommerfeld 
curve. These results have been confirmed by more accurate numerical computations. Zahn et al. (1974) numerically computed these solutions by increasing the number of modes used in the periodic direction to two, and found a nonlinear neutral surface. Herbert $(1976,1977)$ used a spectral collocation technique which employs up to four modes and found the critical two-dimensional Reynolds number of the neutral surface at $R e=2935$ and wavenumber $\alpha=1.323$. These calculations show that the critical Reynolds number is reduced by a factor of 2 compared to linear theory. However, there is still a large discrepancy from the experimentally observed critical Reynolds number of $\approx 1000$. In addition, the results show that even for a calculation with one mode, the results are qualitatively correct. This is surprising because the first nonlinear interaction involves the second harmonic. Further confirmation of the above results has been provided by several groups (e.g. Milinazzo \& Saffman 1985).

In $\$ 2$, we repeat these calculations because we wish to study two-dimensional secondary bifurcations. Because we use a more accurate procedure to determine the critical two-dimensional Reynolds number, there are slight discrepancies between Herbert's results and our own. In addition, we outline the numerical scheme which will be implemented throughout this paper.

The discussion so far has been restricted to steady two-dimensional waves. Orszag \& Patera (1983) found quasi-steady two-dimensional waves well below $R e=2900$. These states eventually decay, but the decay rate is so slow that Orszag \& Patera consider these states effectively steady. A three-dimensional stability analysis is then implemented under the assumption that the variation of the two-dimensional states on the slow timescale can be neglected. As an initial condition for this stability analysis, they use a two-dimensional state which is steady. The three-dimensional perturbations are found to grow explosively if the amplitude of the two-dimensional state is sufficiently large and the decay rate of the two-dimensional state is sufficiently small. Below a Reynolds number of $\approx 1000$, they find that the twodimensional decay rate is too large for three-dimensional disturbances to grow. Based on these results, Orszag \& Patera propose that transition is the threedimensional instability of two-dimensional quasi-steady flows.

It remains of interest, however, to search for steady equilibria at Reynolds numbers below 2900. With the motivation of Saffman's hypothesis, we searched for such steady solutions. In $§ 3$, we study the two-dimensional superharmonic stability of two-dimensional waves. Previously, stability studies have focused on the threedimensional stability of two-dimensional waves. This is due to the observation that three-dimensional instabilities grow on a convective timescale while two-dimensional disturbances grow on a viscous timescale. As transition occurs explosively, it is believed that three-dimensional instabilities initiate transition. Our efforts, however, are aimed at finding bifurcations to two-dimensional states which exist at low Reynolds number. The three-dimensional instabilities of these states would then lead to transition.

It has been demonstrated by Pugh \& Saffman (1988) that the two-dimensional stability of two-dimensional waves depends on the particular stream-function boundary conditions applied at the walls. A Reynolds number can be defined by fixing the spatially averaged pressure gradient to be constant, or by fixing the flux to be constant. In $\S 3$ we rederive these results, concentrating on the correct derivation of stability for constant-pressure disturbances. For constant flux disturbances, we find, in agreement with previous work that the lower branch of the two-dimensional waves is unstable and a stability transition occurs at the limit point. The upper branch, however, does not remain stable. Two different pairs of 
complex-conjugate eigenvalues cross the imaginary axis transversely. These crossings are Hopf bifurcations.

For constant-pressure disturbances, we find that the lower branch is unstable but a stability transition does not occur at the limit point. Instead, another eigenvalue becomes unstable at the limit point. The two unstable eigenvalues merge on the upper branch and subsequently stabilize. The point of stabilization is a Hopf bifurcation. This Hopf bifurcation is not present in the case of constant-flux disturbances. In addition, we find two more Hopf bifurcations on the upper branch, which correspond to the Hopf bifurcations found in constant-flux disturbances. The presence of these various Hopf bifurcations implies the existence of periodic solutions or, in a fixed frame of reference, quasi-periodic solutions.

In $\S 4$, we compute the branches of periodic orbits which emanate from the Hopf bifurcations found in $\$ 3$. Previously, Jiménez (1987) found one of the branches of these solutions using a numerical simulation of two-dimensional dynamics. The Hopf bifurcation for this branch occurs on the upper branch of the neutral surface at a Reynolds number of $\approx 5000$ and $\alpha=1.0$. Jiménez found that the Reynolds number of this branch increases with increasing amplitude. We confirm this result in our calculations. In addition, we calculate several unstable branches which Jiménez could not detect with a simulation of the dynamics. As discussed above, a new Hopf bifurcation appears when the Reynolds number is based on constant pressure. For the Hopf bifurcation which only appears in the case of constant-pressure disturbances, we find that the Reynolds number initially decreases with increasing disturbance amplitude. A limit point, however, is reached before the Reynolds number extends below the critical two-dimensional Reynolds number. For the other branches of periodic orbits, the Reynolds number increases with increasing disturbance amplitude and does not appear to turn back.

Recently Barkley (1990) has made use of the numerical results reported here to derive the form of the amplitude equations which hold near the nose of the neutral surface. In particular he examines a range of stability problems by defining a parameter which interpolates between the constant-pressure and constant-flux results. Coupled with detailed knowledge about the location and criticality of the bifurcation points presented here and by Pugh \& Saffman (1988), he confirms the result that it is not possible to continue the sub-critical Hopf bifurcations beyond the critical two-dimensional Reynolds number. These results depend crucially on the observation (obtained from these numerical results) that no additional bifurcations appear as one follows the path of the sub-critical Hopf bifurcation. From this analysis Barkley provides global bifurcation diagrams for these solutions.

Finally it should be noted that, given the nature of our numerical scheme, it has not been possible to sufficiently refine the resolution so that numerical convergence of the results is unequivocally demonstrated. We have attempted throughout the paper to indicate that while the results are not fully converged, they are qualitatively sensible.

\section{Steady two-dimensional waves}

Consider the two-dimensional Navier-Stokes equations for incompressible flow in the primitive variable formulation:

$$
\frac{\partial u}{\partial t}+(u \cdot \nabla) u=-\frac{1}{\rho} \nabla p+\nu \nabla^{2} u
$$




$$
\boldsymbol{\nabla} \cdot \boldsymbol{u}=\mathbf{0},
$$

where $u=(u, v)$, the velocities in the $x$ - and $y$-directions respectively. We introduce a stream function $\Psi(x, y, t)$ by letting

$$
u=\frac{\partial \Psi}{\partial y}, \quad v=-\frac{\partial \Psi}{\partial x}
$$

With the stream function as defined by (2.3), we transform (2.1)-(2.2) to the equivalent stream function formulation:

$$
\frac{\partial \nabla^{2} \Psi}{\partial t}+\Psi_{y} \nabla^{2} \Psi_{x}-\Psi_{x} \nabla^{2} \Psi_{y}=\nu \nabla^{4} \Psi
$$

where the subscripts denote differentiation with respect to the indicated variable. For flow down a channel, an exact solution to (2.4) is

$$
\Psi=U_{0}\left(y-\frac{y^{3}}{3 h^{2}}\right)
$$

This solution satisfies the no-slip boundary conditions

$$
\left.\begin{array}{l}
v=\frac{\partial \Psi}{\partial y}=0, \\
v=-\frac{\partial \Psi}{\partial x}=0,
\end{array}\right\} y= \pm h
$$

and has a parabolic velocity profile. We call this the basic solution and let $\Psi_{\mathrm{b}} \equiv$ $U_{0}\left(y-y^{3} / 3 h^{2}\right)$.

To find bifurcations to two-dimensional travelling waves, we study the stability of $\Psi_{\mathrm{b}}$ to infinitesimal disturbances. Thus let

$$
\Psi=\Psi_{\mathrm{b}}+\epsilon \phi(y) \mathrm{e}^{\mathrm{i} \alpha(x-\epsilon t)},
$$

where $\alpha$ is the wavenumber and $c=c_{\mathrm{r}}+\mathrm{i} c_{i}$ is the complex phase speed of the disturbance. Substituting (2.6) into (2.4)-(2.5) and scaling lengths by the channel half-width $h$ and velocities by the characteristic velocity $U_{0},(2.4)-(2.5)$ become

$$
\begin{gathered}
\frac{1}{\mathrm{i} \alpha \operatorname{Re}}\left(\mathrm{D}^{2}-\alpha^{2}\right)^{2} \phi=(U-c)\left(\mathrm{D}^{2}-\alpha^{2}\right) \phi-U^{\prime \prime} \phi=0, \\
\alpha \phi=\mathrm{D} \phi=0, \quad y= \pm 1,
\end{gathered}
$$

where $R e=U_{0} h / \nu$ is the Reynolds number, $\mathrm{D}$ represents differentiation with respect to $y, U=1-y^{2}$ is the dimensionless basic velocity, and $U^{\prime \prime}$ denotes the second derivative with respect to $y$ of the basic velocity. Equation (2.7) is the Orr-Sommerfeld equation. For a given wavenumber and Reynolds number, $(2.7)$ and (2.8) constitute an eigenvalue problem for the eigenvalue $c$ and the eigenfunction $\phi(y)$.

Only one eigenfunction of (2.7) is known to have an eigenvalue $c(\alpha, R e)$ whose imaginary part becomes greater than zero in a region of the $(\alpha, R e)$-plane (Drazin \& 
Reid 1981). The curve of points defined by $c_{\mathrm{i}}=0$ is known as the curve of marginal stability. If one holds the wavenumber fixed and increases the Reynolds number in order to pass through the marginal stability curve, the eigenvalue and its complex conjugate cross the imaginary axis transversely. Therefore, the curve of marginal stability also represents a curve of Hopf bifurcation points.

To solve this problem numerically, we impose two additional equations to specify the phase and amplitude of the disturbance. One convenient way is to set

$$
\operatorname{Re} \phi^{\prime \prime}(-1)=c_{1}, \quad \operatorname{Im} \phi^{\prime \prime}(-1)=c_{2},
$$

where $c_{1}$ and $c_{2}$ are constants.

We applied the software package aUTo developed by Doedel \& Kernevez (1985) to solve (2.7)-(2.9). Newton iteration is used to solve the nonlinear system along the solution branch with four interpolating collocation points per mesh point and up to 50 mesh points to accurately resolve the boundary layers formed at high Reynolds numbers. In addition, the mesh was adjusted after every step to minimize the local discretization error.

To compute the marginal curve, an initial guess must be provided for the eigenfunction, eigenvalue, and the other parameters. The eigenvalue spectrum for (2.7) has been derived for $R e \ll 1$ (Drazin \& Reid 1981). Using this spectrum as an initial guess, we continue the solution to the curve where $c_{i}=0$. We found the minimum Reynolds number to be 5772.22. This value compares well with the value computed by Orszag (1971) and Davey, Hocking \& Stewartson (1974).

We next compute steady two-dimensional travelling waves in the streamwise direction with phase speed $c$. Letting $\Psi(x, y, t)=\Psi_{\mathrm{b}}+\psi(\tilde{x}, y)$, where $\tilde{x}=x-c t$, and scaling lengths and velocities as above, $(2.1)$ becomes

$$
(U-c) \nabla^{2} \psi_{x}-\frac{1}{R e} \nabla^{4} \psi-U^{\prime \prime} \psi_{x}+\psi_{y} \nabla^{2} \psi_{x}-\psi_{x} \nabla^{2} \psi_{y}=0 .
$$

Using a spectral decomposition, we set

$$
\psi(x, y)=\sum_{n=-\infty}^{\infty} \hat{\psi}_{n}(y) \mathrm{e}^{\mathrm{i} \alpha n x},
$$

yielding the modal equations

$$
\begin{aligned}
-\frac{1}{R e}\left(S_{x}^{4}+2 S_{x}^{2} \frac{\mathrm{d}^{2}}{\mathrm{~d} y^{2}}+\frac{\mathrm{d}^{4}}{\mathrm{~d} y^{4}}\right) & \hat{\psi}_{n}+(U-c)\left(S_{x}^{2}+\frac{\mathrm{d}^{2}}{\mathrm{~d} y^{2}}\right) S_{x} \hat{\psi}_{n}-U^{\prime \prime} S_{x} \hat{\psi}_{n} \\
& +\frac{\mathrm{d} \hat{\psi}}{\mathrm{d} y} *\left(S_{x}^{2}+\frac{\mathrm{d}^{2}}{\mathrm{~d} y^{2}}\right) S_{x} \hat{\psi}-\left(S_{x} \hat{\psi}\right) *\left(S_{x}^{2}+\frac{\mathrm{d}^{2}}{\mathrm{~d} y^{2}}\right) \frac{\mathrm{d} \hat{\psi}}{\mathrm{d} y}=0,
\end{aligned}
$$

where $S_{x} f_{n}=\mathrm{i} \alpha n f_{n}$ and

$$
f * g=\sum_{m=-\infty}^{\infty} f_{n-m} g_{m}
$$

is the convolution of the two Fourier series. The reality of $\psi(x, y)$ indicates that

$$
\hat{\psi}_{-n}(y)=\hat{\psi}_{n}^{*}(y)
$$

implying that only modes $n \geqslant 0$ need to be determined. 
The modal no-slip boundary conditions are

$$
\begin{aligned}
& \frac{\mathrm{d} \hat{\psi}_{n}}{\mathrm{~d} y}=0, \quad y= \pm 1, \quad n \geqslant 0, \\
& \mathrm{i} \alpha n \hat{\psi}_{n}=0, \quad y= \pm 1, \quad n \geqslant 0 .
\end{aligned}
$$

For $n=0$, the constant mode of $v$ is identically zero and we must specify two additional boundary conditions. As $\psi$ is arbitrary to within a constant, we let

$$
\hat{\psi}_{0}(-1)=0 \text {. }
$$

One boundary condition is still undetermined. This indeterminacy results from an arbitrariness in the choice of $U_{0}$. One possible choice is to define $U_{0}$ such that there is no perturbation to the average flux. The average flux $Q$ is given by

$$
Q=\frac{\alpha h}{2 \pi} \int_{0}^{2 \pi / \alpha} \int_{-1}^{1} u \mathrm{~d} y \mathrm{~d} x .
$$

Using (2.3) with $\Psi=\Psi_{\mathrm{b}}+\psi(x, y)$, we obtain

$$
Q=\left.\left(\Psi_{b}+\hat{\psi}_{0}\right)\right|_{-1} ^{+1}
$$

To permit no perturbation to the average flux, we set

$$
\hat{\psi}_{0}(1)-\hat{\psi}_{0}(-1)=0 \text {. }
$$

Equations (2.17) and (2.14) yield the additional boundary condition

$$
\hat{\psi}_{0}(1)=0 \text {. }
$$

Alternatively, one can define $U_{0}$ such that there is no perturbation to the average pressure gradient. The average pressure gradient $P$ is given by

$$
P=\left.\frac{\nu}{2 h}\left(\Psi_{\mathrm{b}, y y}+\hat{\psi}_{0, y y}\right)\right|_{-1} ^{+1}
$$

To allow no perturbation to the average pressure gradient, we set the additional boundary condition

$$
\hat{\psi}_{0, y y}(1)-\hat{\psi}_{0, y y}(-1)=0 \text {. }
$$

Equations (2.18) and (2.20) represent the two limits of zero average flux perturbation and zero average pressure gradient perturbation. In general, it is possible to take a linear combination of these flows and thus there is a continuous range of boundary conditions. Corresponding to (2.18) and (2.20) are two different Reynolds number $R e_{Q}$ and $R e_{P}$ :

$$
\begin{gathered}
R e_{Q}=\frac{3 Q}{4 \nu} \\
R e_{P}=-\frac{h^{3} P}{2 \nu^{2}}
\end{gathered}
$$

It is important to note that these Reynolds numbers represent different scalings of the problem and not different physics. In fact, using (2.16) and (2.19) one can show that

$$
R e_{P}=\operatorname{Re}_{Q}\left[1-\frac{1}{4}\left[\hat{\psi}_{0 Q, y y}(1)-\hat{\psi}_{0 Q, y y}(-1)\right]\right],
$$

where $\hat{\psi}_{0 Q, y y}$ denotes that the second derivative was calculated with the constant 


\begin{tabular}{cccccccc} 
Parameters & \multicolumn{3}{c}{ Herbert (1976) } & & \multicolumn{3}{c}{ Present } \\
\cline { 2 - 4 } \cline { 7 - 8 } & $N=1$ & $N=2$ & $N=3$ & & $N=1$ & $N=2$ & $N=3$ \\
$\alpha$ & 1.2220 & 1.3130 & 1.3201 & & 1.2222 & 1.3129 & 1.3179 \\
$R e_{P}$ & 2825.56 & 2701.74 & 2911.6 & & 2825.56 & 2701.72 & 2911.36 \\
$c$ & 0.3458 & 0.3663 & 0.3643 & & 0.3458 & 0.3663 & 0.3640
\end{tabular}

TABLE 1. Comparison of phase speeds for two-dimensional travelling waves in plane Poiseuille flow with results of Herbert (1976)

flux boundary condition. For planar Poiseuille flow these two Reynolds numbers are equivalent. In general $R e_{P}=f\left(R e_{Q}, \alpha\right)$ and for two-dimensional waves $R e_{P}>R e_{Q}$ (Saffman 1983).

An additional equation is required to eliminate the arbitrary phase shift in the $x$ direction. If $\hat{\psi}_{n}$ is a solution for a given $c, \hat{\psi}_{n} \mathrm{e}^{\mathrm{i} n \delta \alpha}$ is also a solution where $\delta$ is arbitrary. To eliminate this indeterminacy, we set

$$
\frac{\operatorname{Im} \psi_{1}^{\prime \prime}(-1)}{\operatorname{Re} \psi_{1}^{\prime \prime}(-1)}=\text { constant }
$$

To continue the solution branch of the travelling wave solutions of $(2.10)$ into the nonlinear regime, the amplitude of the nonlinear wave must be defined. One possible choice is related to the disturbance energy:

$$
A_{E}^{2}=\frac{15}{16} \sum_{\substack{n=-N \\ n \neq 0}}^{N} \int_{-1}^{+1}\left|u_{n}(y)\right|^{2}+\left|v_{n}(y)\right|^{2} \mathrm{~d} y
$$

The factor $15 / 16$ is chosen so that the energy of the basic flow is normalized to one.

Truncating (2.12) at $n=N$, we are left to solve a finite system of nonlinear ordinary differential equations (ODE's). We implement the numerical scheme described above and compute solution branches to the resulting nonlinear algebraic system by pseudo-arclength continuation. An initial guess is provided by the eigenfunction calculation described above.

Several authors have calculated the two-dimensional waves discussed in this section. We have repeated the calculation to facilitate the study of two-dimensional secondary bifurcations to be discussed in the later sections. As a check on our calculations, we show that our results are in good agreement with the earlier calculations.

In table 1, we compare our values for the critical two-dimensional Reynolds number of the nonlinear neutral surface, based on constant pressure, to Herbert's (1976) results for $N=1, N=2$ and $N=3$ modes. As shown in the table, there are slight quantitative discrepancies between Herbert's results and our own. These discrepancies can be attributed to the more accurate procedure which was used in our calculation. Herbert (1976) determined the critical two-dimensional Reynolds number by determining the limit point in Reynolds number for each wavenumber. We used a two-parameter continuation of the limit point to determine the critical two-dimensional Reynolds number. Two-parameter continuation of limit points is described in Keller (1988) and is implemented in AUTO. To check our results, we computed the critical Reynolds number at the critical wavenumber given by Herbert (1976) and confirmed his results. 
In summary, we have calculated the marginal stability curve for plane Poiseuille flow. The points on this curve are bifurcation points to two-dimensional travelling waves. We calculated the nonlinear neutral surface which is formed from the solution branches of these waves. The critical two-dimensional Reynolds number of this surface was found to be approximately $2900 \pm 100$ based on constant pressure gradient and $2600 \pm 100$ based on constant flux. Although this reduces the critical Reynolds number from linear theory by a factor of 2 , there is still a large discrepancy from the Reynolds number of $\approx 1000$ found in experiment.

\section{Stability to two-dimensional disturbances}

In this section, we examine the two-dimensional stability of the two-dimensional waves found in plane Poiseuille flow. In particular, we are interested in the stability of these flows to perturbations of the same wavelength as the two-dimensional waves.

In $\$ 1$, no two-dimensional waves were found below a Reynolds number of 2600 (based on flux). This suggests that some other two-dimensional or three-dimensional steady state will be needed to explain the experimentally observed transition Reynolds number of approximately 1000. The search for such flows motivates the work in this section. We study the two-dimensional stability of two-dimensional waves in search of bifurcations to other two-dimensional states which may exist below a Reynolds number of 2600 .

An earlier two-dimensional stability analysis by Pugh \& Saffman (1988) pointed out some interesting features. From considerations put forth by Joseph (1976) and others, Orszag \& Patera (1983) predicted that the lower branch of this surface is unstable to two-dimensional disturbances with a stability transition occurring at the limit point. However, as Pugh \& Saffman (1988) showed numerically, in general this prediction is incorrect. In fact, although neutrally stable eigenmodes are present at the limit point, unstable eigenmodes may also be present. In addition, Pugh \& Saffman (1988) found bifurcations to quasi-periodic solutions on the upper branch. At these bifurcations, there is a change of stability. We have augmented these results by performing a more extensive numerical study and several new bifurcations have been found.

We wish to perturb the steady two-dimensional flows discussed in the previous section. Thus, consider the dimensionless form of the stream-function formulation in a frame of reference moving with velocity $c$ :

$$
\frac{\partial}{\partial t}\left(\nabla^{2} \Psi\right)-\frac{1}{R e} \nabla^{4} \Psi+\left(\Psi_{y}-c\right) \nabla^{2} \Psi_{x}-\Psi_{x} \nabla^{2} \Psi_{y}=0
$$

We use Floquet theory to study the stability of the two-dimensional waves. In a frame of reference in which the travelling waves appear steady, the two-dimensional states can be described by a stream function

$$
\Psi_{2 \mathrm{D}}(\tilde{x}, y)=\Psi_{2 \mathrm{D}}(\tilde{x}+2 \pi / \alpha, y),
$$

where $\alpha$ is the wavenumber of the two-dimensional wave. Then, linear perturbations exist of the form

$$
\Psi(\tilde{x}, y, t)=\Psi_{2 \mathrm{D}}(\tilde{x}, y)+\epsilon \mathrm{e}^{(\mathrm{i} p \tilde{x}+\sigma t)} \sum_{n=-\infty}^{+\infty} \hat{\zeta}_{n}(y) \mathrm{e}^{\mathrm{i} \alpha n \tilde{x}},
$$

where $\sigma$ and $\left\{\hat{\zeta}_{n}\right\}$ are the eigenvalue and eigenfunction respectively and $p$ is the 
subharmonic wavenumber of the disturbance. Note that below we use $x$ for $\tilde{x}$. We take $p=0$, which corresponds to superharmonic disturbances, and

$$
\Psi_{2 \mathrm{D}}(x, y)=\Psi_{\mathrm{b}}+\psi(x, y),
$$

where $\Psi_{\mathrm{b}}$ is the stream function of the basic flow and $\psi(x, y)$ represents a twodimensional secondary flow. Substituting (3.4) into (3.3), equation (3.1) becomes

$$
\begin{aligned}
& -\frac{1}{R e}\left[S_{x}^{4} \hat{\zeta}_{n}+2 S_{x}^{2} \hat{\zeta}_{n, y y}+\hat{\zeta}_{n, y y y y}\right]+S_{x}\left[(U-c)\left(S_{x}^{2} \hat{\zeta}_{n}+\hat{\zeta}_{n, y y}\right)-U^{\prime \prime} \hat{\zeta}_{n}\right] \\
& \quad+\hat{\psi}_{y} *\left[S_{x}\left(S_{x}^{2} \hat{\zeta}+\hat{\zeta}_{y y}\right)\right] \\
& \quad+\hat{\zeta}_{y} *\left[S_{x}\left(S_{x}^{2} \hat{\psi}+\hat{\psi}_{y y}\right)\right]-S_{x} \hat{\psi} *\left(S_{x}^{2} \hat{\zeta}_{y}+\hat{\zeta}_{y y y}\right) \\
& \quad-S_{x} \hat{\zeta} *\left(S_{x}^{2} \hat{\psi}_{y}+\hat{\psi}_{y y y}\right)=\sigma\left(S_{x}^{2} \hat{\zeta}_{n}+\hat{\zeta}_{n, y y}\right),
\end{aligned}
$$

where $U$ is the dimensionless basic velocity and we have used the spectral representation for $\psi(x, y)$. Applying the no-slip boundary conditions at the walls, we have in modal form

$$
\begin{array}{ll}
n \neq 0: & \hat{\zeta}_{n y}( \pm 1)=0 \text { corresponding to } u_{n}( \pm 1)=0 \\
& \hat{\zeta}_{n}( \pm 1)=0 \text { corresponding to } v_{n}( \pm 1)=0 \\
n=0: & \hat{\zeta}_{0 y}( \pm 1)=0 \text { corresponding to } u_{0}( \pm 1)=0 .
\end{array}
$$

For $n=0, v_{0}=0$ is identically satisfied and two additional boundary conditions must be given. As the stream function is arbitrary to within a constant, we let

$$
\hat{\zeta}_{0}(-1)=0 .
$$

The final boundary condition is determined by fixing the parametrization of the problem. Two possible choices are

or

$$
\begin{gathered}
\hat{\zeta}_{0}(+1)-\hat{\zeta}_{0}(-1)=0, \\
\sigma\left[\hat{\zeta}_{0}(+1)-\hat{\zeta}_{0}(-1)\right]=\frac{1}{R e}\left[\hat{\zeta}_{0, y y}(+1)-\hat{\zeta}_{0, y y}(-1)\right],
\end{gathered}
$$

corresponding to the maintenance of constant flux or constant pressure respectively.

To derive the above boundary conditions we consider the dimensionless momentum equation in the $x$-direction :

$$
\frac{\partial u}{\partial t}+u \frac{\partial u}{\partial x}+v \frac{\partial u}{\partial y}=-\frac{\partial p}{\partial x}+\frac{1}{R e} \nabla^{2} u .
$$

Using integration by parts and the continuity equation, the convective terms disappear upon averaging in the $x$-direction and integration over the channel width. The momentum equation, therefore, becomes

$$
\frac{\partial \psi_{0}}{\partial t}(1)-\frac{\partial \psi_{0}}{\partial t}(-1)=-2 P+\frac{1}{R e}\left(\psi_{0, y y}(1)-\psi_{0, y y}(-1)\right),
$$

in terms of the stream function $\psi$. Here $\psi_{0}$ represents the zeroth mode of the Fourier representation for $\psi$, and $P$ is the average pressure gradient. Only the constant mode 
is present for the stream function since we are averaging over $x$. Substituting (3.3) for the stream function and specifying no perturbation to the basic pressure gradient, we derive (3.9) and (2.19). By failing to consider the time derivative in the Navier-Stokes equation, Pugh \& Saffman (1988) stated the constant pressure boundary condition as

$$
\hat{\zeta}_{0, y y}(+1)-\hat{\zeta}_{0, y y}(-1)=0 \text {. }
$$

As can be seen from (3.9), this omission should introduce errors for large Reynolds number, and in fact this will be shown numerically. We implemented the method of spectral collocation (Gottlieb \& Orszag 1977) to solve the system of ODE's and boundary conditions described by (3.5)-(3.9). We let

$$
\hat{\zeta}_{n}(y)=\sum_{k=0}^{N} a_{n k} \tilde{T}_{k}(y),
$$

where $\tilde{T}_{k}(y)$ is a modified Chebyshev polynomial. The polynomials are modified to satisfy the boundary conditions (3.6)-(3.8) identically. In order to satisfy the constraint of constant average flux we set

$$
\tilde{T}_{k}(y)=\left(1-y^{2}\right)^{2} T_{k}(y),
$$

and to satisfy the constraint of constant average pressure gradient

$$
\tilde{T}_{k}(y)=\int_{-1}^{y}\left(1-x^{2}\right) T_{k}(x) \mathrm{d} x
$$

where $T_{k}(y)=\cos k\left(\cos ^{-1} y\right)$. Derivatives of $\hat{\zeta}_{n}(y)$ are computed by differentiating the Chebyshev modes.

Substituting (3.11) into (3.5), it remains to solve a system of algebraic equations for $a_{n k}(n=0, \ldots, N, k=0, \ldots, K)$. With the modifications described by (3.12) and (3.13), the boundary conditions (3.6)-(3.7) are identically satisfied, and only (3.8) or (3.9) must be imposed on the system of equations. Gottlieb \& Orszag (1977) have shown that the maximum error can be approximately minimized by evaluating the equations at the maxima of the $K$ th Chebyshev polynomial. Evaluating at these maxima $\left(\theta_{j}=j \pi / K, y_{j}=\cos \theta_{j}, j=0, \ldots, K\right)$ and truncating (3.5) at a finite number of modes, we only need to solve a discrete generalized eigenvalue problem of the form

$$
\boldsymbol{G a}=\boldsymbol{\sigma B a}
$$

for the complex eigenvalue $\sigma$ and complex eigenvector $\boldsymbol{a}$.

Because large errors were incurred when using standard generalized eigenvalue solvers, we inverted the matrix $\boldsymbol{B}$ and solved the regular eigenvalue problem

$$
\boldsymbol{C a}=\sigma a \text { where } \boldsymbol{C}=\boldsymbol{B}^{-1} \mathbf{G} .
$$

The matrix $C$ is complex if one uses the exponential form of the Fourier series shown in (3.3). Because the secondary flow is real, a real formulation for the matrix $\boldsymbol{C}$ can be derived by writing the Fourier series in trigonometric form. The real formulation reduces the memory requirements of the computation by a factor of 4 . We also note that the necessity for inverting the matrix $B$ in (3.14) motivated the modification of the Chebyshev polynomials described by (3.12)-(3.13). The enforcement of the timeindependent boundary conditions (3.6)-(3.7) on (3.14) would introduce rows of zeros in the matrix $B$ and prevent its inversion.

Calculations were performed for both constant-flux and constant-pressure 


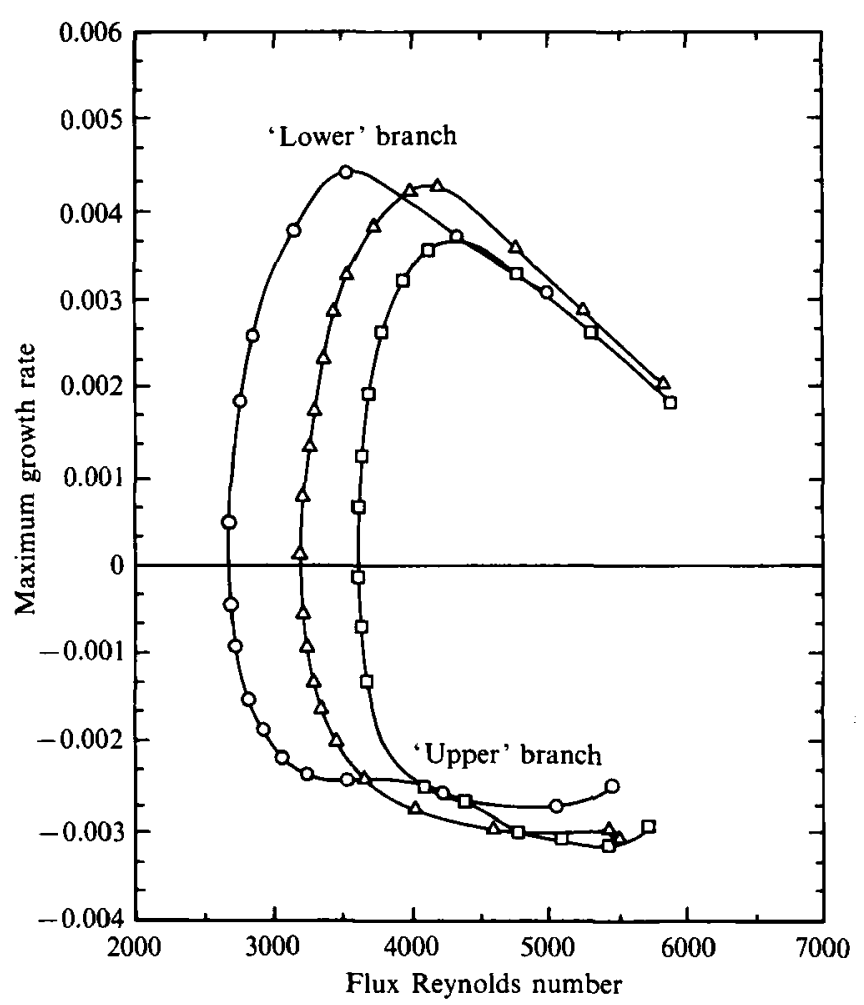

Figure 1. A plot of the maximum growth rate versus flux Reynolds numbers $\left(R e_{Q}\right)$ for twodimensional disturbances of the finite-amplitude travelling waves computed in $§ 2$. Results shown here are for $\alpha=1.1$. Note that the limit point for travelling wave solutions is in this case a point of transition from stability to instability as one traverses the neutral curve from the upper branch through the limit point to the lower branch. Calculations are shown here for $N=1$ (circles), $N=$ 2 (triangles), and $N=3$ (squares) modes with $K=70$ collocation points in the $y$-direction.

disturbances. All of the calculations to be discussed were performed with $\alpha=1.1$. We first consider constant-flux disturbances. In figure 1 we plot the maximum growth rate as a function of Reynolds number for $N=1$ Fourier mode and $K=70$ Chebyshev modes. The most unstable eigenvalue is always real in the range of Reynolds number shown. The lower branch is unstable with a stability transition occurring at the limit point.

At the limit point, there are two neutrally stable eigenvalues. As Pugh \& Saffman (1988) have shown, one eigenvalue is always zero on the two-dimensional wave branch. This eigenvalue corresponds to the trivial phase shift solution of our system. In addition, there is a neutrally stable eigenvalue at the limit point with geometric multiplicity one and algebraic multiplicity two. Although a stability transition occurs at the limit point, the upper branch does not remain stable. As shown in figure 2 , there is a change of stability at $R e=6300$. As the eigenvalue and its complex conjugate cross the imaginary axis transversely, the point of stability transition represents a secondary Hopf bifurcation. The periodic flows emanating from this bifurcation together with the underlying steady waves lead to quasi-periodic solutions. Using a numerical simulation of the two-dimensional dynamics, Jiménez (1987) detected this bifurcation and followed the quasi-periodic flows. In the next section, we will compute these flows using continuation. 

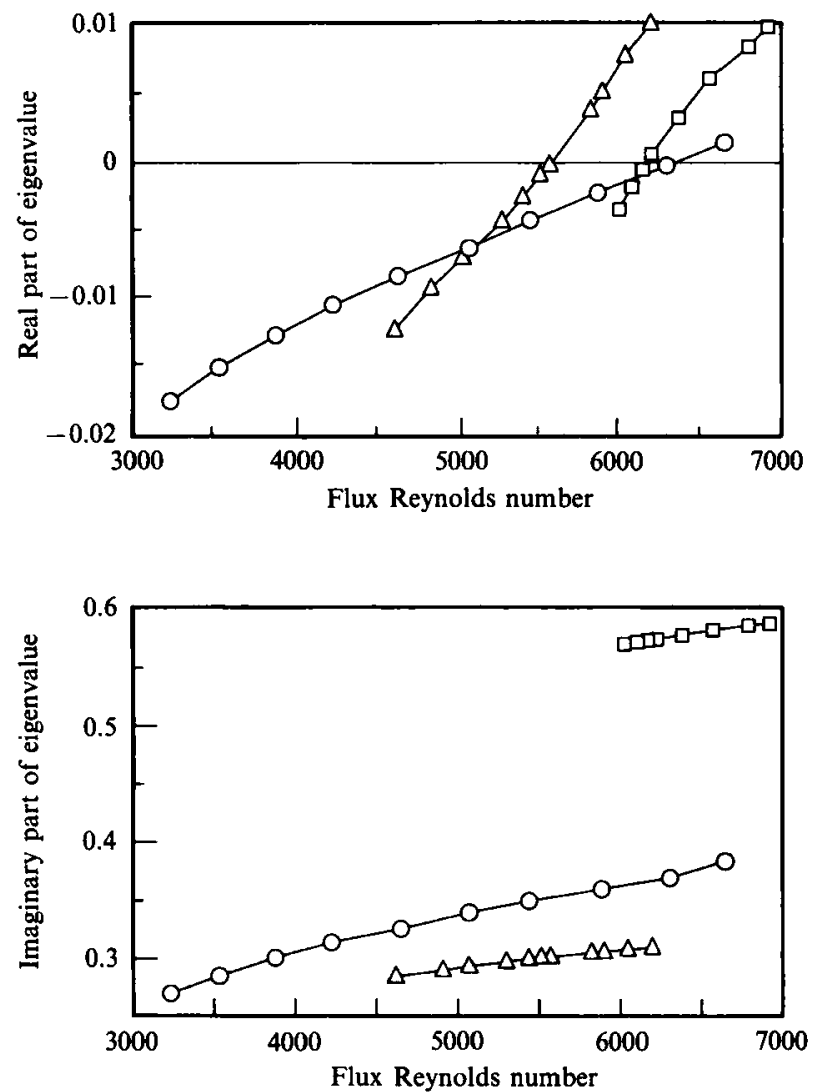

Figure 2. A plot of the real and imaginary parts of a complex eigenvalue corresponding to the stability of solutions on the upper branch versus $R e_{\mathrm{e}}$. The crossing point through zero of the real part of the eigenvalue represents the first Hopf bifurcation on the upper branch. Calculations are shown for $N=1$ (circles), 2 (triangles), and 3 (squares) modes, $\alpha=1.1$ and $K=70$. See also table 2 for numerical values.

In figure 1 we also plot the maximum growth rate as a function of Reynolds number for $N=2$ Fourier modes and $K=70$ Chebyshev modes. As in the calculation with one Fourier mode, the lower branch is unstable with a stability transition occurring at the limit point. However, we now find two Hopf bifurcations occurring on the upper branch. The first Hopf bifurcation occurs at a Reynolds number of 5600 with period 20.6, the second at a Reynolds number of 6125 and with period 18.6. The variation of the real and imaginary parts of the relevant eigenvalue for the second bifurcation is shown in figure 3 . We note that as the upper branch is unstable after the first Hopf bifurcation, a simulation of the dynamics will not detect the second Hopf bifurcation. Results for a calculation with $N=3$ Fourier modes and $K=70$ Chevyshev modes are also shown in figures 1-3. Only a quantitative difference can be seen from the $N=2$ calculation. We repeated these calculations for $N=4$ Fourier modes and again no qualitative difference was seen.

In table 2, we tabulate the period and Reynolds number of the two Hopf bifurcations for $N=1-4$ modes. As can be seen, we do not have quantitative convergence. Jiménez (1987) observed that the location of the first Hopf bifurcation did not settle down until $N=7$. Although this resolution is not attainable with our formulation owing to memory limitations, we do obtain qualitative consistency of 

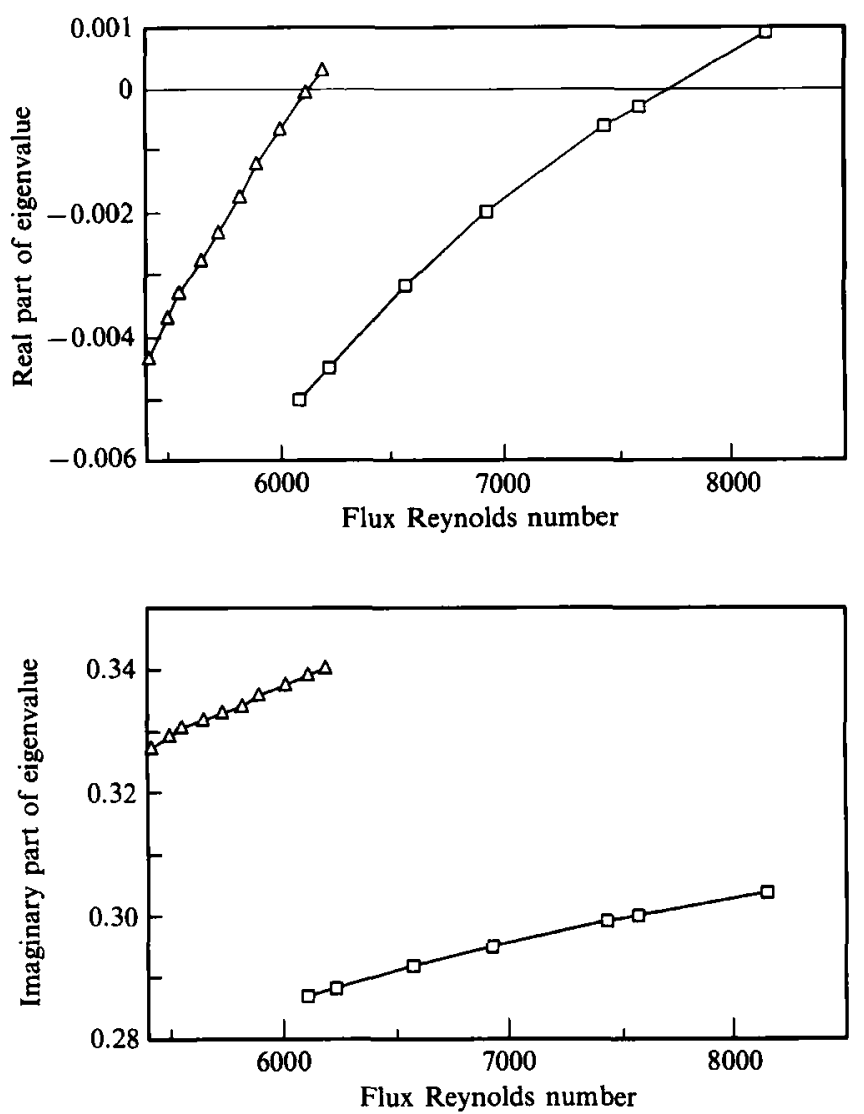

Figure 3. A plot of the real and imaginary parts of a complex eigenvalue corresponding to the stability of solutions on the upper branch versus $R e_{0}$. The crossing point through zero of the real part of the eigenvalue represents the second Hopf bifurcation on the upper branch. Calculations are shown for $N=2$ (triangles) and 3 (squares) modes, $\alpha=1.1$ and $K=70$. See also table 2 for numerical values.

$\begin{array}{cccc}\text { Bifurcation } & N & R e_{Q} & T \\ 1 & 1 & 6300 & 17.5 \\ & 2 & 5600 & 20.6 \\ & 3 & 6250 & 12.5 \\ 2 & 4 & 5875 & 13.4 \\ & 2 & 6125 & 18.6 \\ & 3 & 7750 & 20.73 \\ & 4 & 7500 & 19.75\end{array}$

TABLE 2. Tabulation of the period for Hopf bifurcations from two-dimensional waves in plane Poiseuille flow. Calculations are based on constant flux.

the results, i.e. the Hopf bifurcation does not disappear and the Reynolds number of the bifurcation lies between 5600 and 6300 . Our calculations also confirm some of the quantitative features that Jiménez observed. In particular, the period of the first Hopf bifurcation is decreasing with increasing resolution. Jiménez found that the period of the bifurcation decreased from $\approx 19.3$ to $\approx 14.3$ when increasing the resolution from $N=1$ to $N=7$ modes. For the second Hopf bifurcation, which 


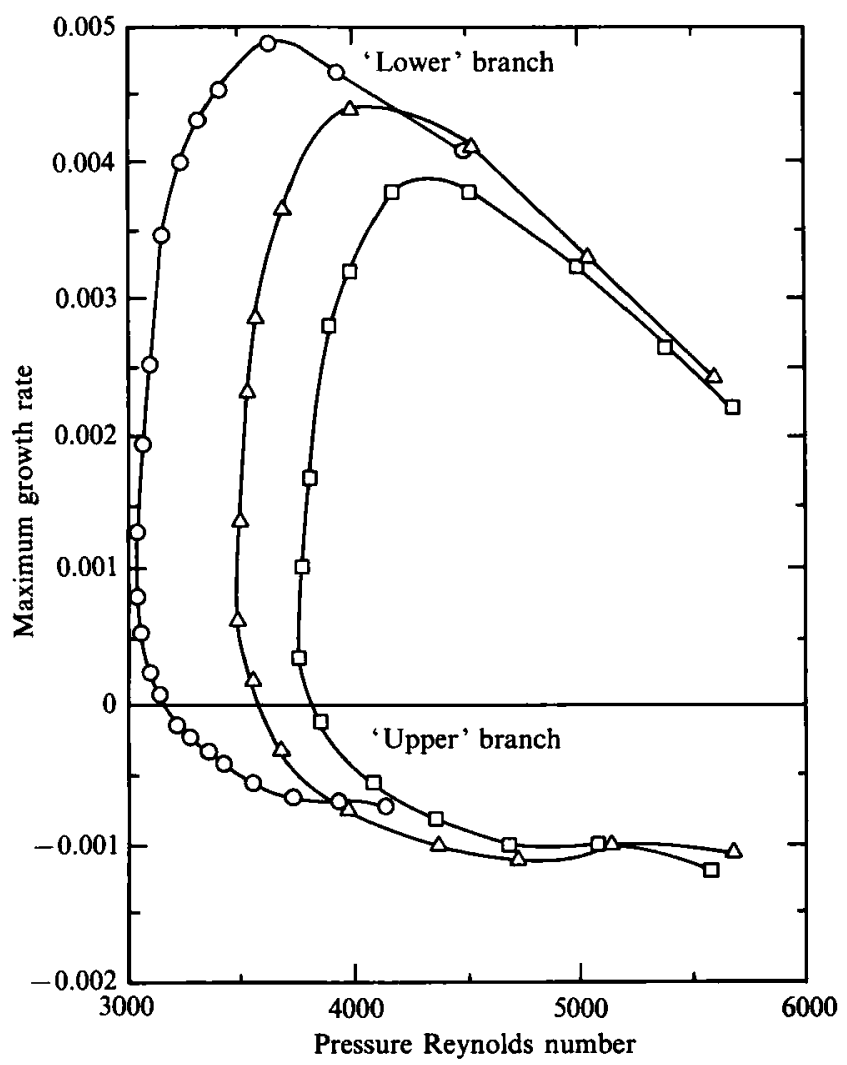

Figure 4. A plot of the maximum growth rate versus pressure Reynolds number $\left(R e_{P}\right)$ for twodimensional disturbances of the finite-amplitude travelling waves computed in $\$ 2$. Results shown here are for $\alpha=1.1$. Note that the limit point for travelling wave solutions is not in this case a point of transition from stability to instability as one traverses the neutral curve from the upper branch through the limit point to the lower branch. The stability transition is due to the different eigenvalue acquiring a positive real part on the upper branch (see also figure 5). Calculations are shown here for $N=1$ (circles), $N=2$ (triangles), and $N=3$ (squares) modes with $K=70$ collocation points in the $y$-direction.

Jiménez could not detect, there is less variation with increased resolution. In $\S 4$, we obtain qualitatively correct results, with low resolution, for the periodic orbits which emanate from these bifurcations. Specifically, we show that we obtain the correct behaviour for the Reynolds number and period with increasing amplitude.

One sees a different stability picture for constant-pressure disturbances. In figure 4, we plot the maximum growth rate as a function of Reynolds number for $N=1-3$ Fourier modes and $K=70$ Chebyshev modes. As in the case of constant-flux disturbances, the lower branch is unstable. A stability transition, however, does not occur at the limit point. As shown in figure 5, the real part of a different eigenvalue passes through zero at the limit point, indicating instability on the upper branch. The two unstable eigenvalues merge on the upper branch and cross the imaginary axis transversely. Thus, we have detected a Hopf bifurcation not present for constant-flux disturbances. In contrast to the results of Pugh \& Saffman (1988), we find that the upper branch does not remain stable. Using $N=1$ modes we find that on the upper branch a complex eigenvalue acquires a positive real part indicating instability at a Reynolds number of 13500 with a period of roughly 36 (see table 3 ). 

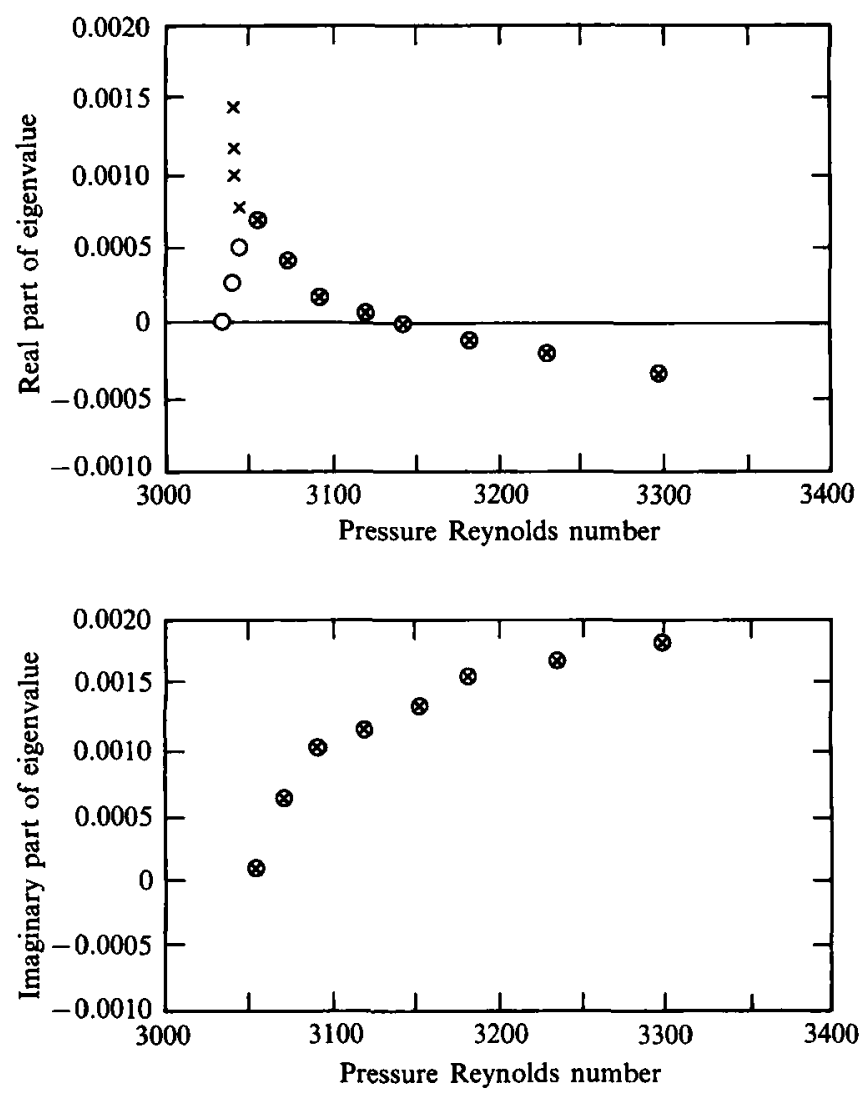

FtaURe 5. A plot of the real and imaginary parts of two eigenvalues as a function of $R e_{P}$. Depicted here is the merger of the real parts of two unstable eigenvalues on the upper branch and the associated Hopf bifurcation as the real part of the merged eigenvalues crosses $\operatorname{Re} \sigma=0$. Calculations are shown with $N=1, K=70$, and $\alpha=1.1$.

$\begin{array}{cccc}\text { Bifurcation } & N & R e_{P} & T \\ 1 & 1 & 3136 & 4833.22 \\ & 2 & 3630 & 4742.32 \\ & 3 & 3800 & 4935.43 \\ 2 & 4 & 3775 & 4875.63 \\ & 1 & 13500 & 36 \\ & 2 & 9400 & 35.5 \\ 3 & 3 & 9675 & 17.65 \\ & 4 & 9592 & 16.54 \\ & 2 & 13000 & 34.0 \\ & 3 & 12775 & 33.76 \\ & 4 & 12960 & 33.85\end{array}$

TaBLE 3. Tabulation of the period for Hopf bifurcations from two-dimensional waves in plane Poiseuille flow. Calculations are based on constant pressure.

The point of stability transition is a Hopf bifurcation and corresponds to the Hopf bifurcation detected in the calculations for constant-flux disturbances. This bifurcation was not detected by Pugh \& Saffman (1988) owing to their use of a slightly different boundary condition to enforce the constraint of constant pressure. 

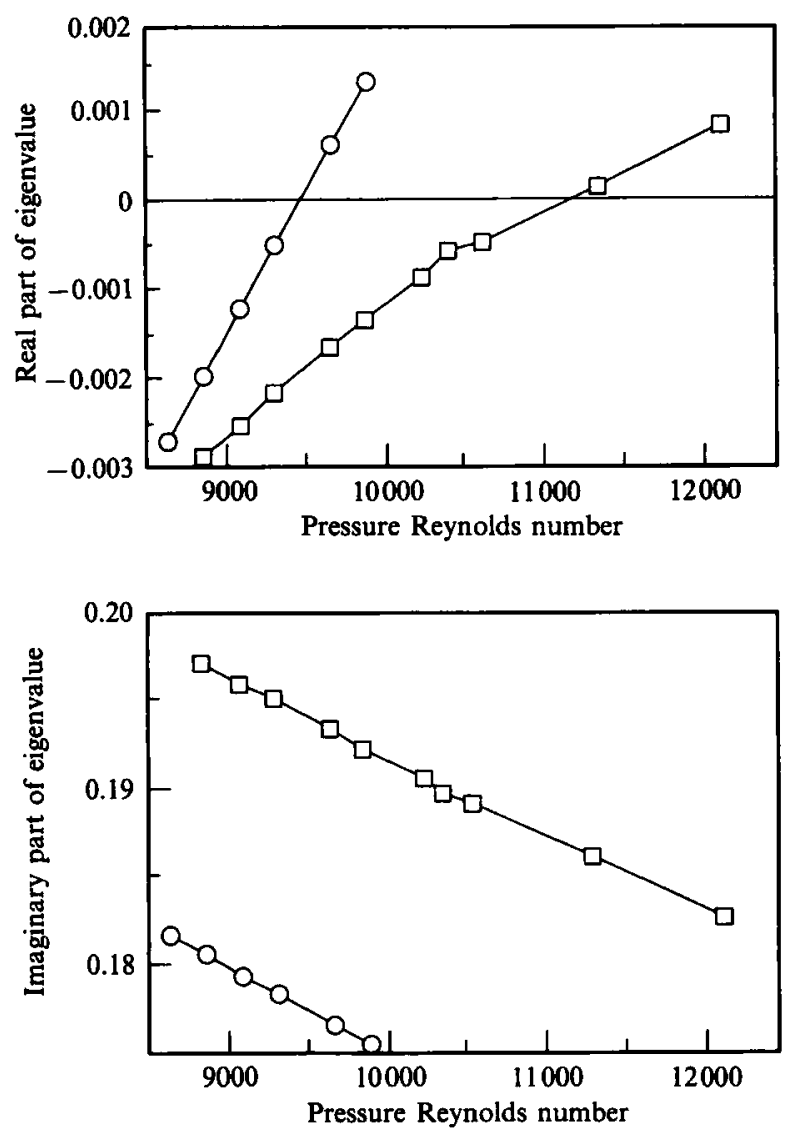

Figure 6. A plot of the real and imaginary parts of two complex eigenvalues corresponding to the stability of solutions on the upper branch versus $R e_{p}$. The crossing points through zero of the real part of the eigenvalue represent the second (circles) and third (squares) Hopf bifurcation on the upper branch. Calculations are shown for $N=2$ modes, $\alpha=1.1$ and $K=70$. See also table 3 for numerical values.

As can be seen from (3.8) and (3.9), for large Reynolds number the constant-flux and constant-pressure boundary conditions are equivalent. In fact, at the Hopf bifurcation the constant-pressure and constant-flux Reynolds number are related by the scaling described in equation (2.23). The use of (3.10) also explains the quantitative difference between their results and our own.

In figures 4-6, we include the results obtained with $N=2$ Fourier modes and $K=$ 70 Chebyshev modes. The results are qualitatively similar to the $N=1$ calculation except that we now detect three Hopf bifurcations on the upper branch. The first Hopf bifurcation corresponds to a transition from instability to stability, and the second occurs at a Reynolds number of 9400 with period 35.5 and corresponds to the Hopf bifurcation detected in the $N=1$ calculation. The upper branch is unstable after this Hopf bifurcation. The third is new and occurs at a Reynolds number of 13000 with period 34. The appearance of a new Hopf bifurcation with $N=2$ Fourier modes corresponds to the results obtained for constant-flux disturbances. This is to be expected as the constant-flux and constant-pressure boundary conditions are equivalent for large Reynolds number.

In figure 4 we also plot the maximum growth rate for a calculation with $N=3$ 
Fourier modes and $K=70$ Chebyshev modes. Only a quantitative change is seen from the $N=2$ calculation, and the three Hopf bifurcations persist. The calculations were repeated for $N=4$ Fourier modes and again only a quantitative change was seen. In table 3, we tabulate the period and Reynolds number for the three Hopf bifurcations on the upper branch. As in the case of constant-flux disturbances, we do not obtain quantitative convergence for the second Hopf bifurcation.

In summary, we have examined the stability of two-dimensional waves to twodimensional superharmonic disturbances. For constant-flux disturbances, the lower branch is unstable with a stability transition occurring at the limit point. In addition, there are two Hopf bifurcations occurring on the upper branch. For constant-pressure disturbances the lower branch is unstable but a stability transition does not occur at the limit point. Instead, the real part of another eigenvalue vanishes at the limit point and becomes positive on the upper branch. The two unstable eigenvalues merge and stabilize; the point of stabilization is a Hopf bifurcation. In addition there are two other Hopf bifurcations on the upper branch. As in the constant-flux disturbances, these Hopf bifurcations correspond to different pairs of eigenvalues which acquire positive real parts on the upper branch. In the next section, continuation is used to compute these bifurcating flows at finite amplitude relative to the two-dimensional waves.

\section{Quasi-periodic solutions}

In this section, we study several branches of quasi-periodic solutions which bifurcate from the two-dimensional travelling waves. We look for quasi-periodic solutions by solving boundary-value problems via continuation and Newton's method. This approach allows us to calculate both stable and unstable solution branches. Because we are studying the transition regime both stable and unstable solutions are of interest.

We again consider the dimensionless form of the two-dimensional Navier-Stokes equation corresponding to a frame of reference moving with speed $c$ and look for solutions of the form

$$
\Psi(x, y, t)=\Psi_{b}+\psi=\Psi_{\mathrm{b}}+\sum_{n=-\infty}^{\infty} \sum_{m=-\infty}^{\infty} \mathrm{e}^{\mathrm{i} m \omega t} \mathrm{e}^{\mathrm{i} n \alpha x} \hat{\psi}_{m n}(y),
$$

where $\omega$ is the frequency introduced by the quasi-periodic flow and $\Psi_{\mathrm{b}}$ is the stream function of the basic flow. Substituting (4.1) into (3.1), we derive a modal equation for each $\hat{\psi}_{m n}$ similar in form to (3.2). Because $\Psi(x, y, t)$ is real, we require

$$
\hat{\psi}_{m,-n}=\hat{\psi}_{-m, n}^{*}
$$

This implies that we only need to solve for the modes $m \geqslant 0$ (for all $n$ ). The modal no-slip boundary conditions become

$$
\left.\begin{array}{cccc}
\frac{\mathrm{d} \hat{\psi}_{m n}}{\mathrm{~d} y}=0 & \text { at } y= \pm 1, \quad m \geqslant 0 & \forall n, \\
\mathrm{i} \alpha n \hat{\psi}_{m n}=0 & \text { at } y= \pm 1, \quad m \geqslant 0 & \forall n \neq 0 .
\end{array}\right\}
$$

For $n=0$, 
is identically satisfied, and we must specify $2(M+1)$ additional boundary conditions. As the stream function is arbitrary up to a function of time, we set

$$
\psi(y=-1)=0 \text {. }
$$

Applying (4.4) at every instant of time, we derive the $(M+1)$ boundary conditions

$$
\hat{\psi}_{m 0}(-1)=0, \quad m \geqslant 0 .
$$

The final $(M+1)$ boundary conditions are determined by fixing the parametrization of the problem. One possible parametrization is to disallow any perturbation to the spatially averaged flux. To disallow perturbations to the flux $Q$, we set

$$
\left[\sum_{m=-\infty}^{\infty} \hat{\psi}_{m 0} \mathrm{e}^{\mathrm{i} m \omega t}\right]_{-1}^{+1}=0
$$

Applying (4.6), together with (4.5), at every instant of time, we derive the $(M+1)$ additional boundary conditions

$$
\hat{\psi}_{m 0}(1)=0, \quad m=0, \ldots, M
$$

Alternatively, one can disallow any perturbations to the spatially averaged pressure gradient. The spatially averaged pressure gradient is

$$
P=\frac{\nu}{2 h}\left[\Psi_{\mathrm{b}, y y}+\sum_{m=-\infty}^{\infty} \hat{\psi}_{m 0, y y} \mathrm{e}^{\mathrm{i} m \omega t}\right]_{-1}^{+1}-\left[\sum_{m=-\infty}^{\infty} \mathrm{i} m \omega \hat{\psi}_{m 0} \mathrm{e}^{\mathrm{i} m \omega t}\right]_{-1}^{+1} .
$$

To disallow perturbations to $P$, we set

$$
\sum_{m=-\infty}^{\infty} \mathrm{e}^{\mathrm{i} m \omega t}\left(\frac{\nu}{2 h} \hat{\psi}_{m 0, y y}-\mathrm{i} m \omega \hat{\psi}_{m 0}\right)_{-1}^{+1}=0 .
$$

Applying (4.9) at every instant of time, we derive the $(M+1)$ additional boundary conditions

$$
\frac{1}{R e}\left[\hat{\psi}_{m 0, y y}(1)-\hat{\psi}_{m 0, y y}(-1)\right]=\mathrm{i} m \omega\left[\hat{\psi}_{m 0}(1)-\psi_{m 0}(-1)\right]
$$

As in the case of two-dimensional waves, the two boundary conditions define two Reynolds numbers $R e_{P}$ and $R e_{Q}$ given by (2.21) and (2.22).

To solve the system described by (4.1)-(4.5) with the additional boundary condition (4.7) or (4.10), we must impose two additional equations to eliminate the arbitrariness in the phase corresponding to translations in $x$ and $t$. Specifically, if $\hat{\psi}_{m n}$ is a solution so is $\hat{\psi}_{m n} \exp (\mathrm{i} m \delta \omega) \exp (\mathrm{i} n \beta \alpha)$. To eliminate the arbitrariness in the phase, we set

$$
\frac{\operatorname{Re}\left[\hat{\psi}_{01}^{\prime \prime}(-1)\right]}{\operatorname{Im}\left[\hat{\psi}_{01}^{\prime \prime}(-1)\right]}=c_{1}, \quad \frac{\operatorname{Re}\left[\hat{\psi}_{11}^{\prime \prime}(-1)\right]}{\operatorname{Im}\left[\hat{\psi}_{11}^{\prime \prime}(-1)\right]}=c_{2}
$$

where $c_{1}$ and $c_{2}$ are constants. We also introduce an amplitude $A$, to continue into the nonlinear regime:

$$
A=\left(\sum_{m=-\infty}^{\infty} \sum_{n=-\infty}^{\infty} \hat{\psi}_{m n}^{2}\right)^{\frac{1}{2}}
$$

Truncating (4.3) at a finite number of modes, it remains to solve a nonlinear system of ODE's with the additional equations (4.11)-(4.12) and the appropriate 


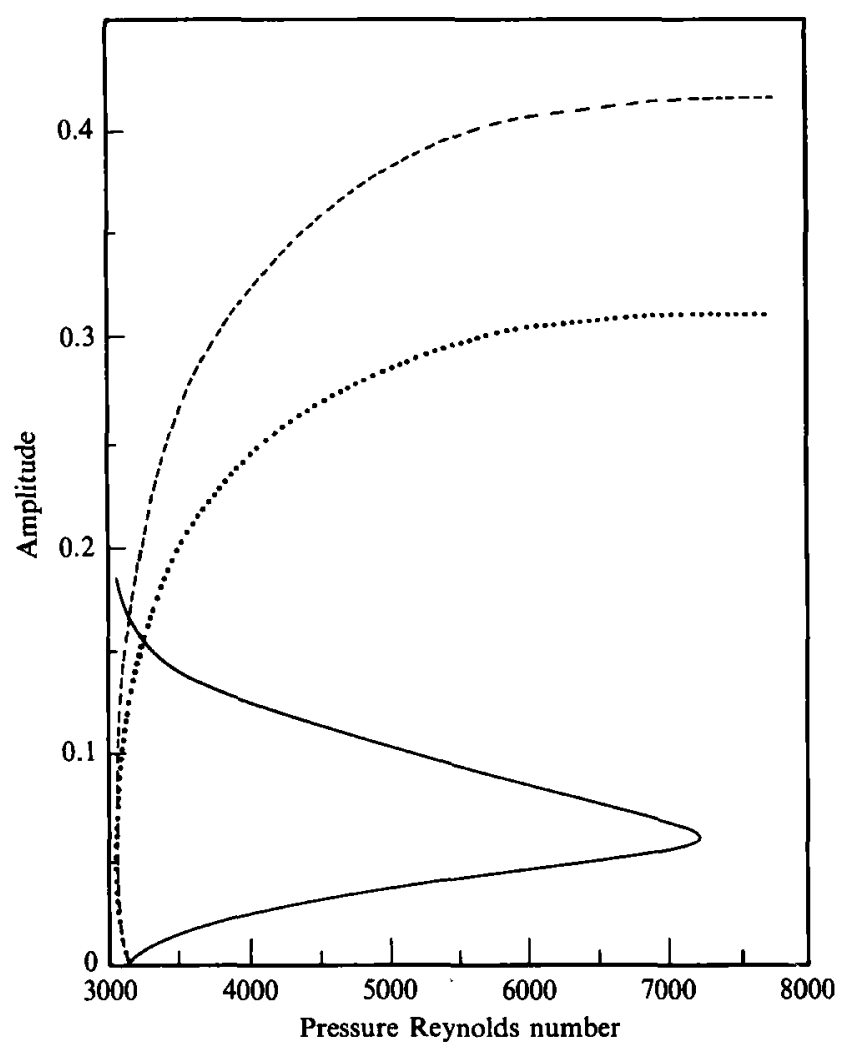

Frgure 7. A plot of the amplitude as a function of Reynolds number based on pressure $R e_{P}$ for quasi-periodic solutions which originate from the Hopf bifurcations discussed in §3. Calculations are shown for $N=1, M=1$ (solid line), $N=1, M=2$ (dashed line), and $N=1, M=3$ (dotted line). All calculations are performed with $K=70$ at a wavenumber $\alpha=1.1$. Note that the calculation for $M=1$ is inadequately resolved and produces qualitatively incorrect results when compared with calculations at higher resolution.

boundary conditions. We implemented the discretization method described in $\S 2$ to solve this system and used arclength continuation to compute the solution branches. An initial guess for these branches is provided by the eigenvectors found in the stability analysis described in $\S 3$.

We first consider the Hopf bifurcations found in constant-pressure disturbances. In §3, we showed that a Hopf bifurcation occurs on the upper branch when the real parts of a pair of complex-conjugate eigenvalues merge and then become negative (see figure 4). In figure 7 we plot amplitude versus Reynolds number for the quasiperiodic solutions which originate from that Hopf bifurcation. The calculations shown use $N=1$ modes in $x, M=1$ to $M=3$ modes in $t$ and $\alpha=1.1$. As shown in figure 7 , for the $M=1$ mode calculation the Reynolds number increases until reaching a limit point at $\mathbf{7 4 0 0}$. The Reynolds number then decreases and the branch of quasi-periodic solutions terminates on the two-dimensional wave branch at a Reynolds number of 3100 . However, as can be seen, the use of only $M=1$ mode yields ill-resolved results. In figure 7, we also plot amplitude versus Reynolds number for $N=1$ and $M=2$ modes. For this calculation, the Reynolds number decreases and reaches a limit point of 3100 . The same qualitative picture is seen for $M=3$ modes. We repeated these calcualtions for $N=2$ and $M=4$ modes and again no qualitative 

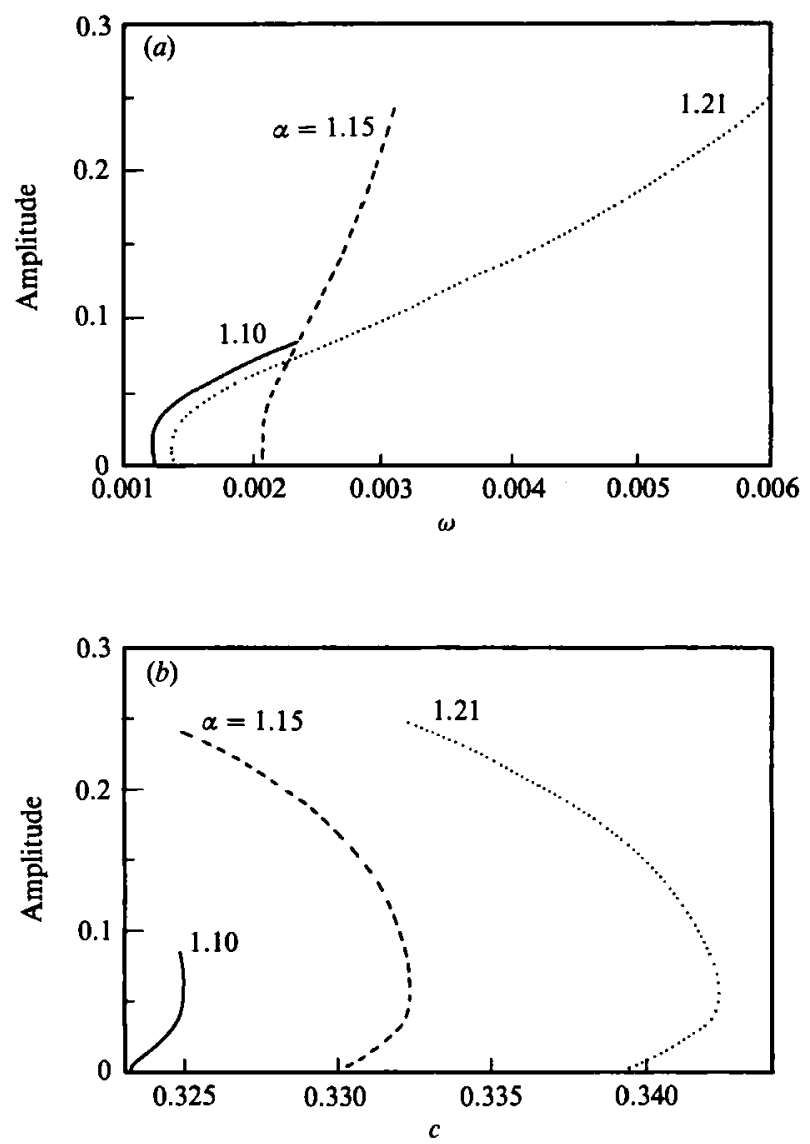

Figure 8. The variation of $(a)$ the frequency $\omega$ and $(b)$ phase speed $c$ with amplitude for quasiperiodic solutions. Calculations are based on constant pressure and are shown for $\alpha=1.10,1.15$, 1.21. Results are shown for resolution $N=1, M=2, K=70$.

change was seen. In figure 8 , we show how $c$ and $\omega$ vary on the branch for various values of $\alpha$. In figure 9 we display the variation of the amplitude versus the Reynolds number for quasi-periodic solutions with $\alpha=1.15$ and $\alpha=1.21$.

In figure 10 we display contours of constant vorticity for a quasi-periodic flow with Reynolds number of 3056 and $\alpha=1.1$. The contours are shown for $y \in[-1,0.78], x$ $\epsilon[0,2 \pi / \alpha]$, and $t \in[0, T]$ where $T=2 \pi / \omega$. As can be seen from the figure, the effect of the modulation in time is to wobble the high-vorticity regions from left to right in $x$. The basic form of the flow appears unaffected.

As the Reynolds number initially decreases with increasing amplitude, the periodic orbits emanating from the Hopf bifurcation are stable (Marsden \& McCracken 1976). One of the eigenvalues, however, will go through zero at the limit point of the periodic orbits. In addition, the branch may become unstable before the limit point. Because of the large memory requirements, we did not implement a Floquet analysis to determine when the branch becomes unstable.

Recently, Barkley (1990) has argued that the branch of quasi-periodic orbits could extend below the critical two-dimensional Reynolds number only if certain events occurred. For example, a secondary bifurcation would have to occur before the limit point of the two-dimensional waves. From our computations we see that the branch 


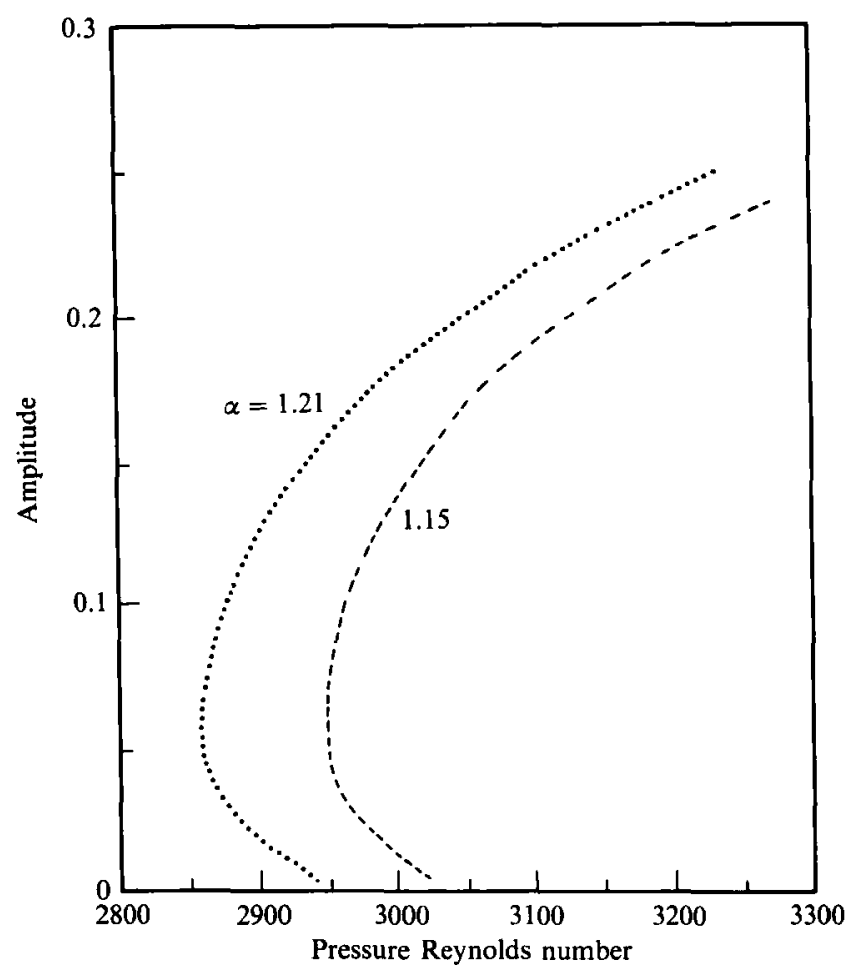

Figure 9. The variation of the amplitude with Reynolds number $R e_{P}$ for quasi-periodic solutions. Calculations are based on constant pressure and are shown for $\alpha=1.15,1.21$. Results are shown for resolution $N=1, M=2, K=70$. Note that both sets of solutions possess limit points, indicating that there is a minimum Reynolds number for existence of these flows.

of quasi-periodic orbits reaches a limit point above the limit point of the twodimensional waves. This scenario has been shown by Barkley to be an acceptable picture in phase space.

It was hoped that this branch of quasi-periodic solutions would extend below a Reynolds number of 2600 (based on constant flux) but for all the wavenumbers studied, however, no such branches were found. The critical Reynolds number of the two-dimensional waves is the envelope for the quasi-periodic solutions. As pointed out above, we did not implement a Floquet analysis of these orbits. The existence of a second Hopf bifurcation or a period-doubling bifurcation cannot be ruled out. Even if such bifurcations were found, however, the large amount of computer memory needed to calculate the solution branches by this approach makes such calculations impractical.

We next discuss the other Hopf bifurcations found on the neutral surface. For both constant-flux and constant-pressure disturbances, two Hopf bifurcations were found on the upper branch. Our calculations showed that all the quasi-periodic solution branches which bifurcate from these Hopf bifurcations are qualitatively similar. Therefore we present a typical solution branch which is representative of these results.

In figure 11, we plot amplitude vs. Reynolds number for the branch of quasiperiodic solutions which bifurcates from the first Hopf bifurcation shown in figure 2 . The calculation shown uses $N=2$ modes in $x$ and $M=2$ modes in $t$. This resolution was found to be adequate for these calculations. As shown in figure 11, the Reynolds 

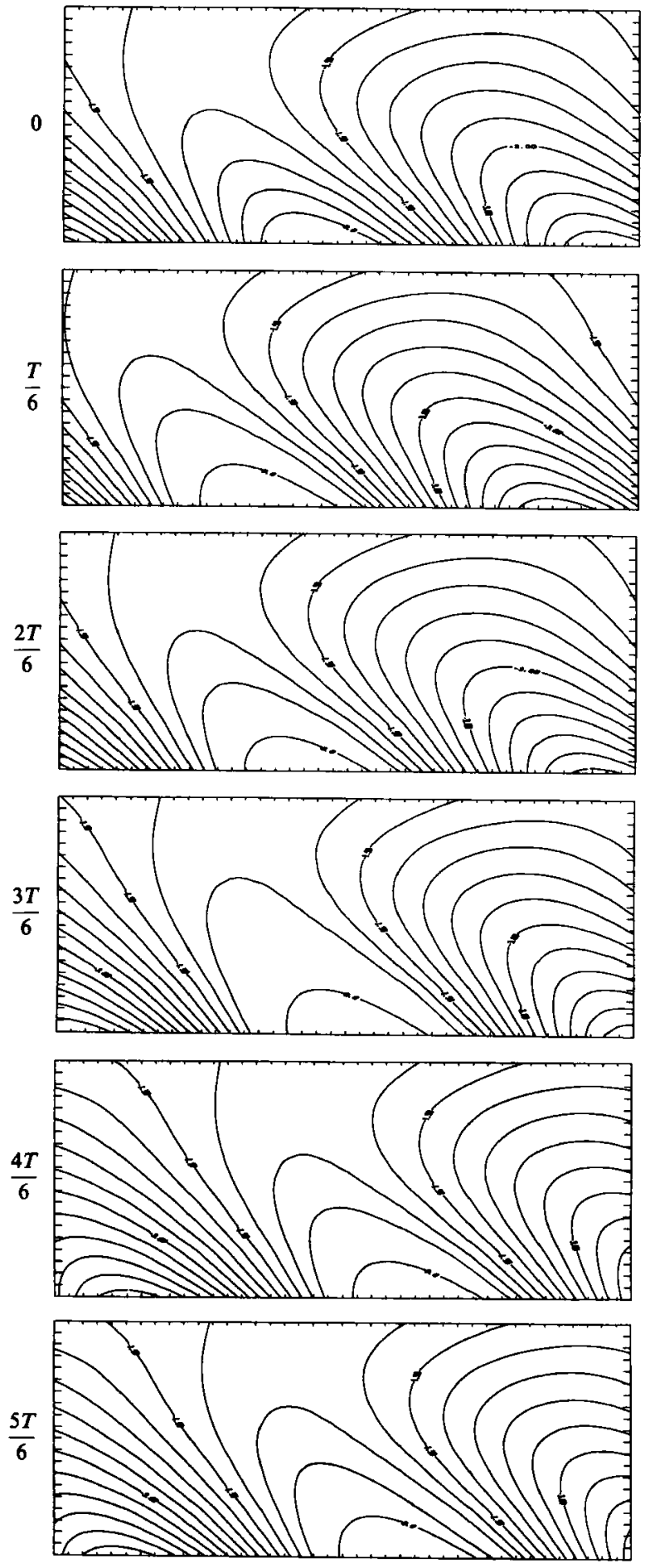

Froure 10. Contours of constant vorticity for quasi-periodic flows. Plots are shown for $t=0$ tc $t=5 T / 6$ in steps of $T / 6$ where $T=2 \pi / \omega$. Contours are computed over the intervals $-1<y<0.78$ and $0<x<2 \pi / \alpha$ with $R e_{P}=3056$, and $\alpha=1.1$. The period $T=4932$. The direction of the basic flow is from left to right. 


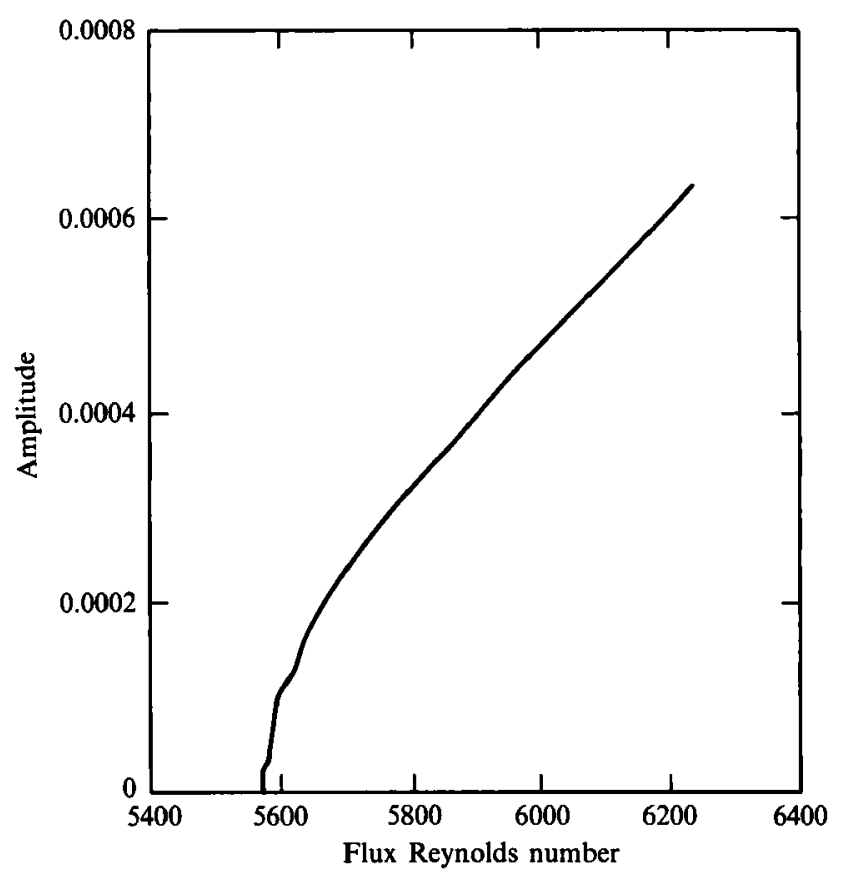

Figure 11. The variation of the amplitude with Reynolds number $R e_{Q}$ for quasi-periodic solutions. Calculations are based on constant flux and are shown for $\alpha=1$.1. Results are shown for resolution $N=2, M=2, K=70$.

number increases with increasing amplitude. As the steady waves are stable before and unstable after the Hopf bifurcation, the branch of quasi-periodic solutions are locally stable to two-dimensional disturbances. To the extent allowed by our numerical calculations these results confirm the calculations by Jiménez (1987). With our steady formulation, we obtain qualitatively similar results, i.e. the Reynolds number increases with increasing amplitude and the period of the orbits decreases with increasing amplitude (ef. figure 12). A quantitative comparison cannot be made since Jiménez used many more modes. As in the case of two-dimensional waves, however, we find that only a few modes are needed to give qualitative agreement.

We also calculated the branch of quasi-periodic solutions which bifurcate from the second Hopf bifurcation which occurs on the upper branch. In addition, we calculated the branch of quasi-periodic orbits which bifurcate from these two Hopf bifurcations for constant-pressure disturbances. As discussed above, all of the branches are qualitatively similar, i.e. the Reynolds number increases with increasing amplitude.

In figure 13, we display contours of constant vorticity for a Reynolds number of $5940, \alpha=1.1, y \in[-1,0.78], t \in[0, T]$, and $x \in[0,2 \pi / \omega]$. As can be seen from the figure, the effect of the modulation in time is to oscillate vertically the regions of high vorticity, again in agreement with the observations of Jiménez (1987). This effect is not as noticeable in figure 10 and 13 as it is in the work of Jiménez owing to the limited resolution of our approach. Jiménez also noted that the vertical oscillation of vorticity is reminiscent of the 'bursting' of vorticity seen in boundary layers. In addition, the timescale of these solutions and of the bursting is of the same order of the three-dimensional flow. 

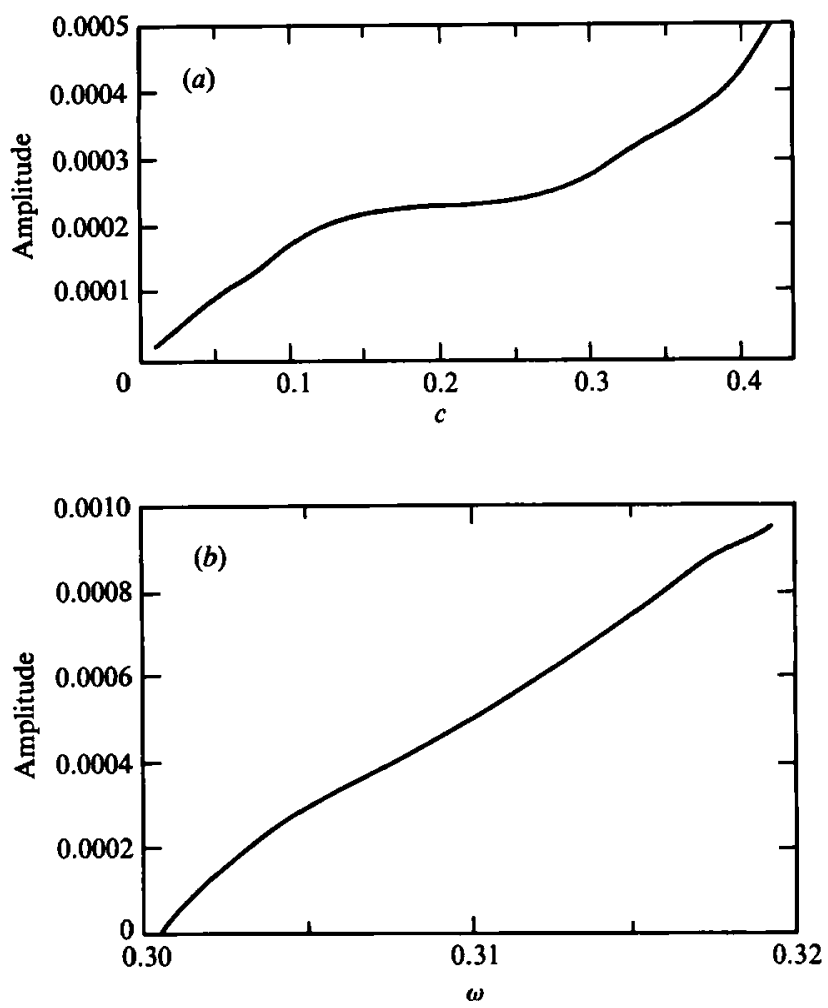

Figure 12. The variation of $(a)$ the frequency $\omega$ and $(b)$ phase speed $c$ with amplitude for quasiperiodic solutions. Calculations are based on constant flux and are shown for $\alpha=1.10$. Results are shown for resolution $N=1, M=2, K=70$.

To summarize this section, we have calculated the branches of quasi-periodic orbits which bifurcate from the two-dimensional waves. For both constant-pressure and constant-flux disturbances, there are two branches of quasi-periodic solutions which bifurcate from the upper branch of the two-dimensional waves. For these branches, we found that the Reynolds number increases with increasing amplitude. Thus the quasi-periodic orbits are stable to two-dimensional disturbances. In addition, the timescale of these orbits is of the same order as three-dimensional flows, and they exhibit phenomena which are reminiscent of 'bursting'. We have also calculated the branch of quasi-periodic orbits which only exists for constant-pressure disturbances. For this branch, we found that the Reynolds number first decreases with increasing amplitude. A limit point, however, is reached above the critical Reynolds number of the two-dimensional waves.

\section{Conclusions}

It is clear that there exist a wide variety of finite-amplitude flows which are bifurcations of plane Poiseuille flow. In this paper we have repeated and confirmed the calculations of travelling waves performed by Zahn, Herbert, and others. We have shown that linear stability analysis of these finite-amplitude states leads to additional bifurcations which take the form of pulsations of vorticity in the boundary layer. Unfortunately, none of these flows exist at Reynolds numbers below the limiting value for existence of travelling waves of permanent form. Thus, while 

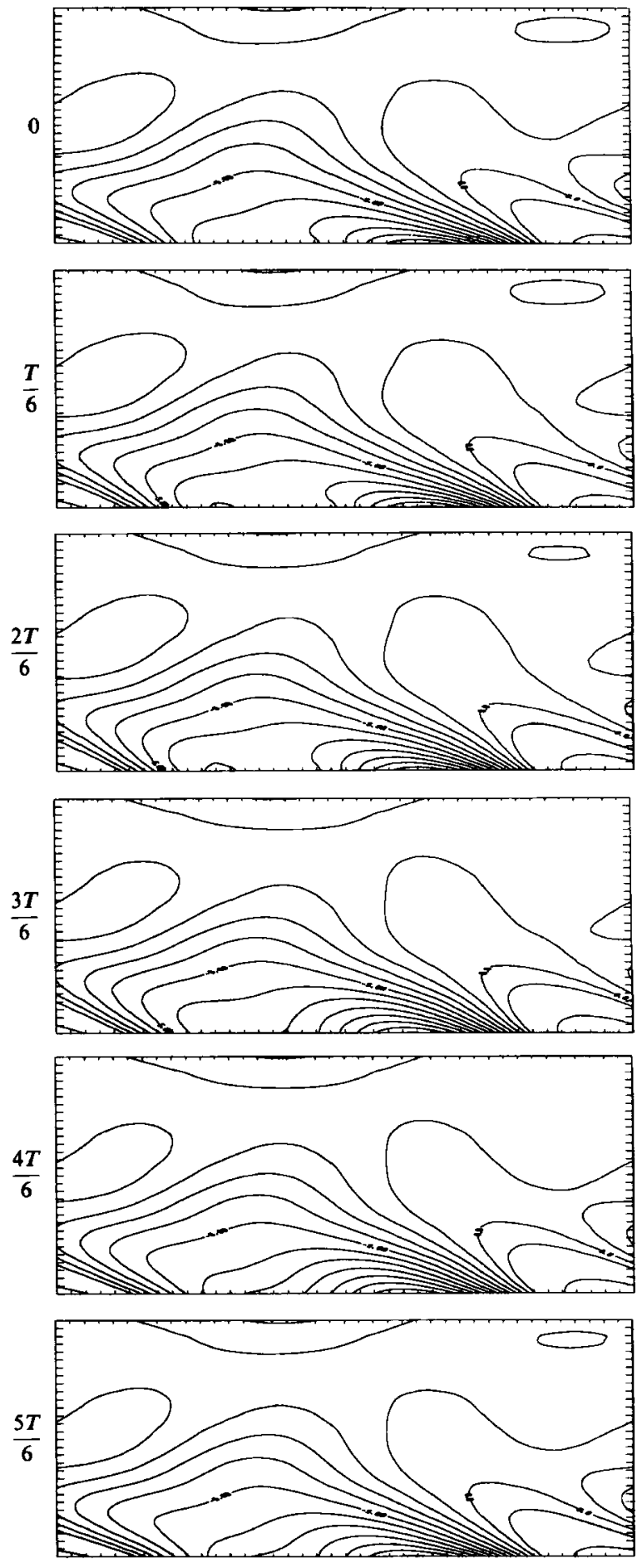

Figure 13. Contours of constant vorticity for quasi-periodic flows. Plots are shown for $t=0$ to $t=5 T / 6$ in steps of $T / 6$ where $T=2 \pi / \omega$. Contours are computed over the intervals $-1<y<0.78$ and $0<x<2 \pi / \alpha$ with $R e_{Q}=5940$, and $\alpha=1.1$. The period $T=20.48$. The direction of the basic Poiseuille flow is from left to right. 
the fluid mechanics of these new solutions is interesting in itself, it fails to provide a basic vortical state to examine transition at the experimentally observed Reynolds numbers close to 1000 . There may of course exist other bifurcations which have more complex structure. One possibility is to examine three-dimensional travelling waves. Goldshtik, Lifshitz \& Stern (1983) have examined such solutions and their initial results indicate that such three-dimensional waves may exist at Reynolds number as low as 1000. Pugh \& Saffman (1988), however, were unable to confirm the existence of these waves. We have also examined certain families of steady three-dimensional waves and this work is described in a subsequent paper.

One problem which plagues all such computations is the need to invert large systems of equations in the application of Newton's method. Using this approach, it is generally impossible to compute flows with more than a few modes in the transverse direction once the vertical direction is resolved. It is for this reason that our calculations display poor convergence. In contrast, dynamic simulations of such flows routinely utilize on the order of $10^{6}$ degrees of freedom. Recently, Tuckerman (1989) has applied an iterative technique to the computation of certain convective flows. This technique has the attractive feature that it only requires factorization of matrices arising from the linear viscous term of the equations and this can be accomplished efficiently even when the number of degrees of freedom is large. While the efficacy of this method for shear flows has not been determined such a technique would allow us to explore steady solutions with the same level of complexity (and similar levels of convergence) as is achieved through dynamic simulation.

We wish to acknowledge helpful discussions with Philip Saffman and Dwight Barkley. This work was supported by the Department of Energy, Office of Energy Sciences (DE-AS03-76ER-72012), Applied Mathematical Sciences (KC-07-01-01).

\section{REFERENCES}

Alavyoon, F., Henningson, D. S. \& Alfredsson, P. H. 1986 Turbulent spots in plane Poiseuille flow - flow visualization. Phys. Fluids 29, 1328-1331.

BARKLEY, D. 1990 Theory and predictions for finite-amplitude waves in two-dimensional plane Poiseuille flow. Phys. Fluids A 2, 955-970.

Carlson, D. R., Widnall, S. E. \& Peeters, M. F. 1982 A flow visualization study of transition in plane Poiseuille flow. J. Fluid Mech. 121, 487-505.

Chen, T. S. \& Joseph, D. D. 1973 Subcritical bifurcation of plane Poiseuille flow. J. Fluid Mech. 58, 337-351.

Davey, A., Hocknna, L. M. \& Stewartson, K. 1974 On the nonlinear evolution of threedimensional disturbances in plane Poiseuille flow. J. Fluid Mech. 63, 529-536.

Doedel, E. J. \& Kernevez, J. D. 1985 Software for continuation and bifurcation problems. Applied Mathematics Tech. Rep., Caltech.

Drazin, P. \& ReId, W. H. 1981 Hydrodynamic Stability. Cambridge University Press.

Goldshtik, M. A., Lifshitz, E. M. \& Shtern, V. N. 1983 The transition Reynolds number for a plane channel, Akad. Nauk. SSSR, Dokl., 273, 75-79.

Gottlieb, D. \& Orszag, S. A. 1977 Numerical Analysis of Spectral Methods: Theory and Application. SIAM-CBMS.

Herbert, Th. 1976 Periodic secondary motions in a plane channel In Proc. Fifth Intl Conf. on Numerical Methods in Fluid Dynamics. Lecture Notes in Physics, vol. 59, p. 235. Springer.

Herbert, Th. 1977 Finite amplitude stability of plane parallel flows. AGARD CP-224, pp. 3-1 10.

Jimḱnez, J. 1987 Bifurcation and bursting in two-dimensional Poiseuille flow. Phys. Fluids 30, 3644-3646.

JosEph, D. D. 1976 Stability of Fluid Motions. Springer. 
KeLler, H. B. 1988 Numerical Methods in Bifurcation Problems. Springer.

Kiм, J. 1983 On the structure of wall-bounded turbulent flows. Phys. Fluids 26, 2088-2097.

KLEISER, L. 1982 Spectral simulations in laminar-turbulent transition in plane Poiseuille flow and comparison with experiments. In Proc. 8th Intl Conf. on Numerical Methods in Fluid Dynamics, Aachen (ed. E. Krause). Lecture Notes in Physics, vol. 170, p. 280. Springer.

Marsden, J. E. \& McCracken, M. 1976 The Hopf Bifurcation and Its Applications. Applied Mathematical Sciences, vol. 19, Springer.

Meksyn, D. \& StuART, J. T. 1951 Stability of viscous motion between parallel surfaces for finite disturbances. Proc. R. Soc. Lond. A 208, 517-526.

Milinazzo, F. \& Saffman, P. G. 1985 Finite amplitude waves in plane viscous shear flows. J. Fluid Mech. 160, 281-295.

Nishioka, M. \& AsaI, M. 1985 Some observations of the subcritical transition in plane Poiseuille flow. J. Fluid Mech. 150, 441-450.

Noether, F. 1921 Das Turbulenzproblem. Z. Angew. Math. Mech. 1, 125-138, 218-219.

Orszag, S. A. 1971 Accurate solution of the Orr-Sommerfeld stability equation. J. Fluid Mech. $50,689-703$.

Orszag, S. A. \& Kells, L. C. 1980 Transition to turbulence in plane Poiseuille flow and plane Couette flow. J. Fluid Mech. 96, 159-206.

Orszag, S. A. \& Patera, A. T. 1983 Secondary instability of wall-bounded shear flows. J. Fluid Mech. 128, 347-385.

Pugh, J. D. \& Saffman, P. G. 1988 Two-dimensional subharmonic stability of finite amplitude waves in plane Poiseuille flow. J. Fluid Mech. 194, 295-308.

REICHARDT, H. 1956 Ửber die Geschwindigkeitsverteilung in einer geradlinigen turbulenen Coutteströmung. Z. Angew. Math. Mech. 36, 454.

Saffman, P. G. 1983 Vortices, stability and turbulence. Ann. NY Acad. Sci. 404, 12-24.

TUCKERman, L. S. 1989 Steady-state solving via Stokes preconditioning: recursion relations for elliptic operators. In Proc. Eleventh Intl Conf. on Numerical Methods in Fluid Dynamics (ed. D. L. Dowyer, M. Y. Hussaini \& R. G. Voight). Springer.

Zahn, J. P., Toomre, J., SPIegel, E. A. \& Gough, D. O. 1974 Nonlinear cellular motions in plane Poiseuille channel flow. J. Fluid Mech. 64, 319-345. 